\title{
QT Interval Prolongation and Atypical Proarrhythmia: Monomorphic Ventricular Tachycardia with Trimebutine
}

\author{
Michele Schiariti ${ }^{1}$, Angela Saladini ${ }^{2}$, Attilio Placanica ${ }^{1}$, Marta Saolini ${ }^{1}$ and Paolo E. Puddu ${ }^{*}, 3$ \\ IDepartment of the Heart and Great Vessels "Attilio Reale", University of Rome "La Sapienza", Rome, Italy \\ ${ }^{2}$ Department of Cardiothoracic Surgery and Cardiology, Sant'Anna Hospital, Catanzaro, Italy \\ ${ }^{3}$ UOC Biotechnologies Applied to Cardiovascular Diseases, University of Rome "La Sapienza", Rome, Italy
}

\begin{abstract}
A 59-year old woman was admitted at emergency for palpitation and dizziness. Medication history showed trimebutine $450 \mathrm{mg}$ daily, because of meteorism, increased to $450 \mathrm{mg}$ TID a week earlier. At admittance, sustained monomorphic ventricular tachycardia was interrupted by $100 \mathrm{mg}$ intravenous lidocaine and a largely prolonged QTc (523 $\pm 12 \mathrm{~ms}$ ) was seen. Discontinuation of trimebutine achieved normalisation of QTc $(420 \pm 10 \mathrm{~ms}, \mathrm{p}<0.001)$.

This is the first report in man to illustrate a probable proarrhythmic action of trimebutine. A weak inhibitory effect on both rapid and slow components of the delayed rectifier in guinea-pig ventricular myocytes calls for further investigations in human myocardial tissues. Trimebutine inhibition of $\mathrm{Na}^{+}$and $\mathrm{Ca}^{++}$channels in cardiac tissues of rabbits and guinea-pigs also call for further studies in human myocardial tissues.
\end{abstract}

Keywords: Trimebutine, proarrhythmia, prokinetic agents, QT prolongation, ventricular tachycardia, delayed rectifier $\mathrm{K}^{+}$ current.

QT interval prolongation is a well known cause of arrhythmia which may be also accounted for by a large variety of drugs affecting action potential duration (APD) in myocardial tissues [1-3]. Some class III agents and other drugs, particularly in guinea-pig hearts [4], by affecting separate components of the delayed rectifier $\mathrm{K}^{+}$current [respectively rapid, $\mathrm{I}(\mathrm{Kr})$ and/or slow, I(Ks) components], may prolong APD and QT interval which may interfere with their anti-arrhythmic capabilities or even cause arrhythmias $[5,6]$. These effects were seen with azimilide or combined dofetilide and HMR 1556 [5] and with the prokinetic agent cisapride, the latter inhibiting $\mathrm{I}(\mathrm{Kr})$ with an $\mathrm{IC}_{50}$ of 15 $\mathrm{nmol} / \mathrm{L}$ which is about $1 / 3$ to $1 / 15$ of the therapeutic levels [6]. Thus several cardiac [1-3] and non-cardiac [7] drugs have a hidden pro-arrhythmic danger accounting for prolonged QT in patients who may later present with lifethreatening arrhythmias, including both monomorphic ventricular tachycardia (VT) [8-11] and, more frequently, torsade de pointes (TdP). Among non-cardiac agents, a strong case was made years ago with common histamine $\mathrm{H}_{1}$ receptor antagonists such as the non sedating agent terfenadine, which prompted important changes acted by regulatory Agencies in pre-registration requirements [2, 12].

There are several commonly used drugs to regulate motility in the gastrointestinal tract of which cisapride [1316] has long been reported to provoke potentially lifethreatening arrhythmias including monomorphic VT and

*Address correspondence to this author at the Dipartimento del Cuore e Grossi Vasi "Attilio Reale", UOC Biotecnologie Applicate alle Malattie Cardiovascolari, Università degli Studi di Roma "La Sapienza", Viale del Policlinico, 155, Rome 00161, Italy; Tel: +39.06.4455291; Fax: +39.06.4441600; E-mail: paoloemilio.puddu@uniroma1.it
TdP. We describe here the occurrence of QT interval prolongation and monomorphic VT during oral administration of the prokinetic agent trimebutine (brand name: Debridat). Although a depressant effect on sinus node pacemaker activity of the rabbit [17] and a weak inhibitory effect on both $\mathrm{I}(\mathrm{Kr})$ and $\mathrm{I}(\mathrm{Ks})$ in guinea-pig ventricular myocytes [18] were reported with trimebutine, this is the first report in man to illustrate a proarrhythmic action, probably due to toxicity. On the other hand, similar to a previously reported case of a patient with human immunodeficiency virus infection and clarithromycin administration, it is illustrated that drug-induced long QT may be in conjunction with monomorphic VT [9], which might be less unusual than previously thought $[1,7,8,10$, $11]$.

\section{CASE REPORT}

A 59-year old woman presented at emergency with palpitation and dizziness. She had a previous history of mitral valve replacement for rheumatic disease ten years earlier and had chronic atrial fibrillation (AF). A more recent angiography showed normal coronary arteries and good overall contractility and no ventricular hypertrophy. She had no family history of syncope, sudden death, or known long QT syndrome and was followed-up in our outpatient clinic where digoxin, diuretics and acenocumarol were prescribed. At last visit, two weeks earlier, she was in NYHA class I and apart from AF the ECG was unremarkable. At emergency, the ECG showed monomorphic sustained VT (Fig. 1) which was interrupted by the intravenous administration of $100 \mathrm{mg}$ lidocaine. The ECG recorded immediately after VT interruption showed a prolonged QTc at $523 \pm 12 \mathrm{~ms}$ by Bazett's formula (Fig. 2). Thereafter, continuous monitoring 


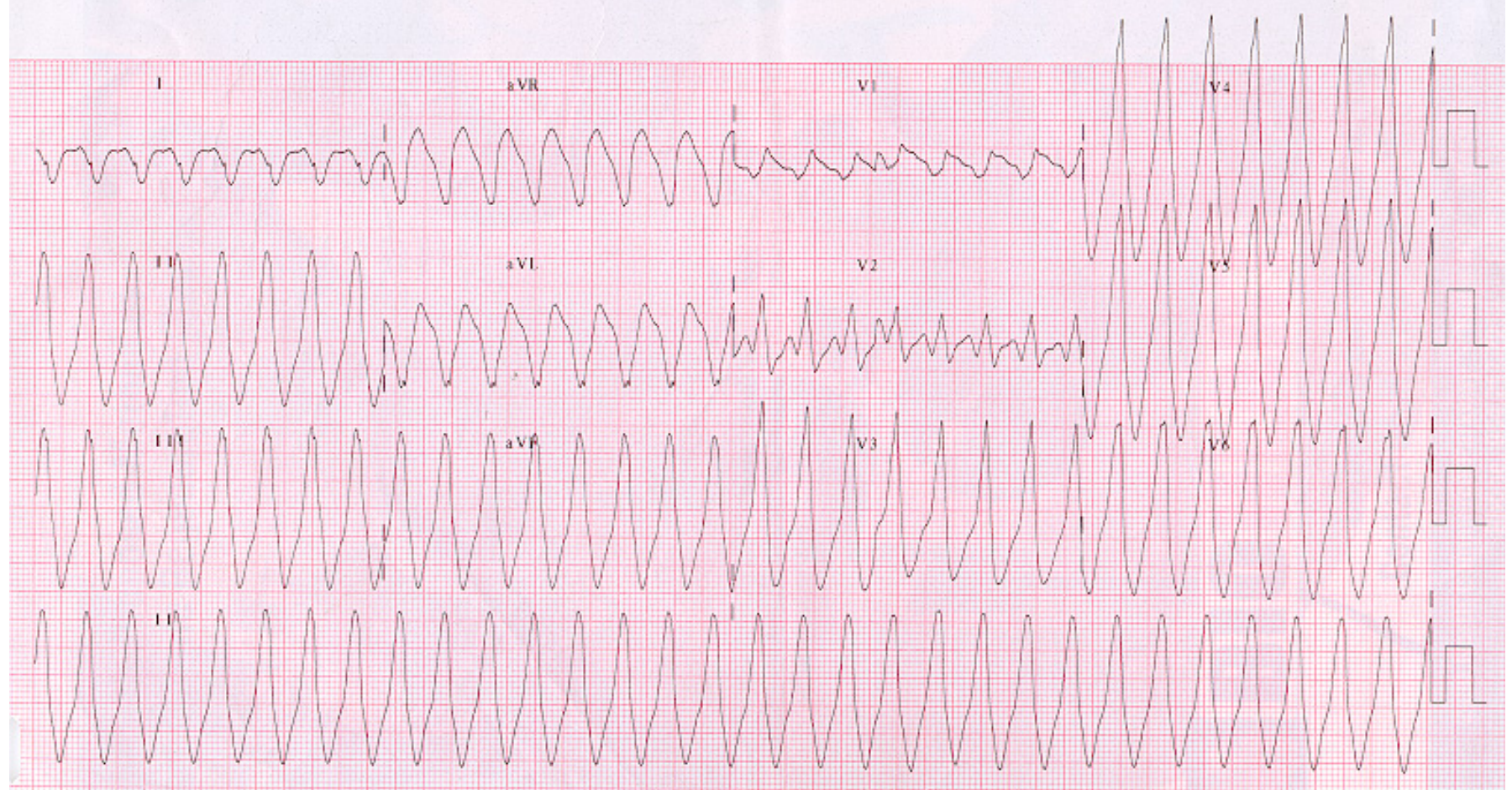

Fig. (1). Standard ECG recorded at patient's admittance showed monomorphic sustained VT.

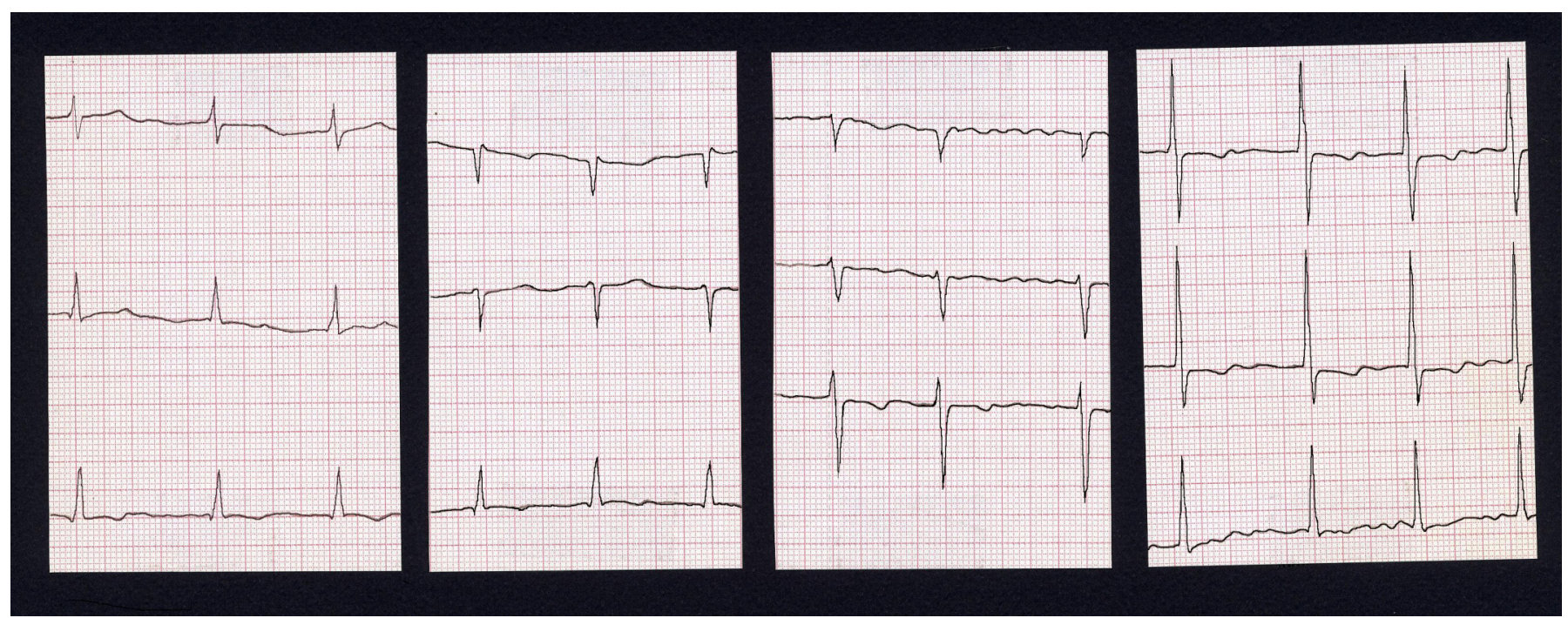

Fig. (2). After intravenous administration of $100 \mathrm{mg}$ lidocaine monomorhic sustained VT was interrupted. This ECG was recorded immediately after VT interruption and shows atrial fibrillation (which was chronic in this patient). QTe obtained with Bazett's [2] formula was extremely prolonged at $523 \mathrm{~ms}$ (average QT $=480 \mathrm{~ms}$; average RR $=840 \mathrm{~ms}$ ). Average QTc, measured using several complexes was $523 \pm 12 \mathrm{~ms}$.

of ECG showed premature beats and short runs of VT (data not shown).

Serum electrolytes, creatinine clearance and digoxin plasma levels were normal. At careful medication history, it was ascertained that a general practitioner prescribed trimebutine, $450 \mathrm{mg}$ daily (3 pills of $150 \mathrm{mg}$ ), because of meteorism. However, the patient decided on her own, because of the worsening of meteorism, to increase trimebutine to $450 \mathrm{mg}$ TID a week earlier. Discontinuation of trimebutine, after VT interruption, achieved normalisation of QTc which was $420 \pm 10 \mathrm{~ms}$, a highly significant $(p<0.001)$ difference (Fig. 3). Signal averaging tests were not performed.

\section{DISCUSSION}

The actions of trimebutine [3,4,5-trimethoxybenzoic acid 2(dimethylamino)-2-phenylbutylester] on the gastrointestinal tract are mediated via (a) an agonist effect on peripheral $\mu$-, $\mathrm{k}$ - and $\delta$ - opiate receptors and (b) release of gastrointestinal peptides such as motilin and modulation of the release of other peptides, including vasoactive intestinal peptide, gastrin and glucagon. Trimebutine accelerates gastric 


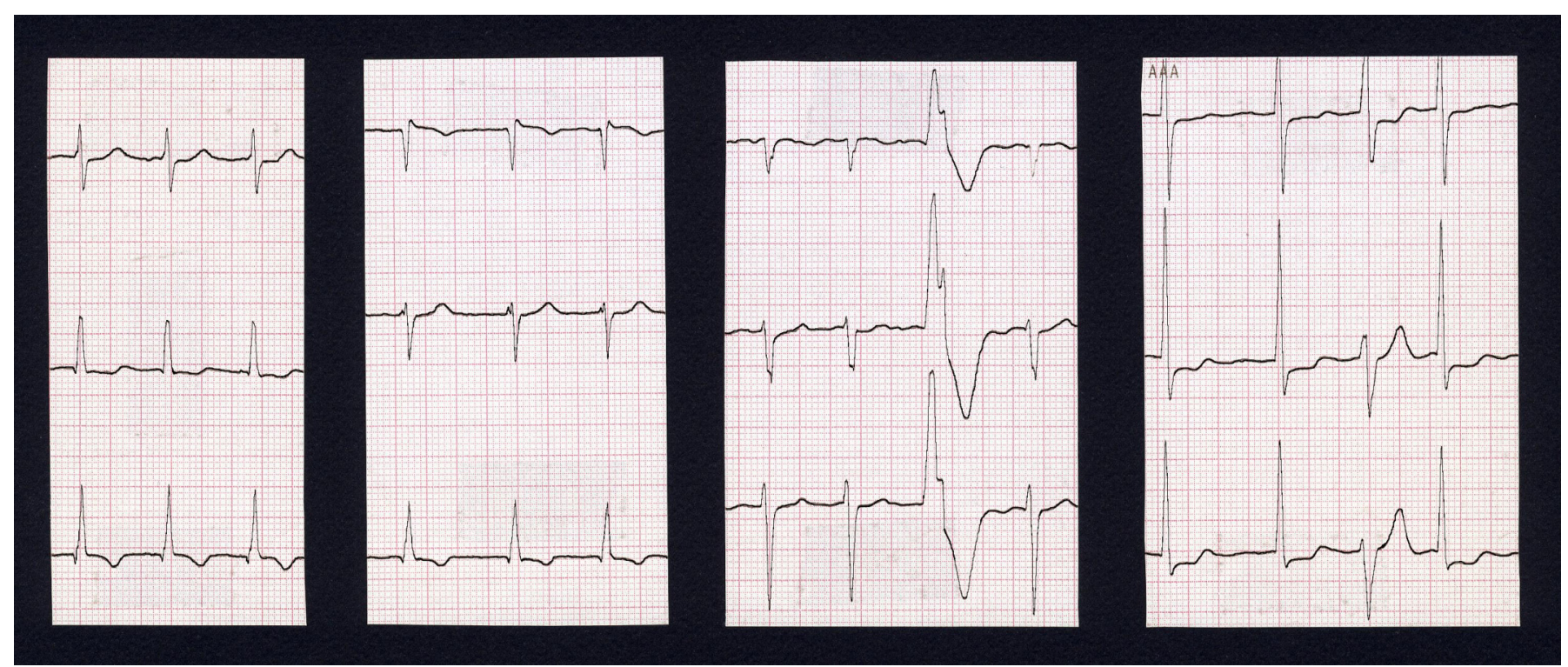

Fig. (3). Two days after admittance and trimebutine interruption another ECG showed persistent atrial fibrillation, premature ventricular beats and normalization of QTc $(424 \mathrm{~ms})$ always obtained by Bazett's [2] formula (average QT $=360 \mathrm{~ms}$; average RR $=720 \mathrm{~ms}$ ). When average QTc, measured using several complexes in this post-discontinuing trimebutine ECG (QTc $=420 \pm 10 \mathrm{~ms})$ was compared with that of Fig. (2), statistically significant shortening was concluded $(\mathrm{p}<0.001)$.

emptying, induces premature phase III of the migrating motor complex in the intestine and modulates the contractile activity of the colon. Clinically, trimebutine has proved to be effective in the treatment of both acute and chronic abdominal pain in patients with functional bowel disorders, especially irritable bowel syndrome, at doses ranging from 300 to $600 \mathrm{mg} /$ day. It is also effective in children presenting with abdominal pain [19]. However, few electrophysiological investigations were carried out with trimebutine $[17,18,20]$.

Cisapride (maleate) is an orally administered prokinetic agent used in the treatment of dyspepsia, diabetic gastroparesis, and gastroesophageal reflux disease, similarly to trimebutine or itopride hydrochloride. Although cisapride is useful and generally well tolerated, QT interval prolongation, TdP and sudden death have been reported in several cases [13-15]. Cisapride-induced arrhythmias are mediated by excessive prolongation of APD caused by blockade of $\mathrm{I}(\mathrm{Kr})$, leading to early afterdepolarization and $\mathrm{TdP}$, but it is possible that pharmacokinetic mechanisms attributed to increased cisapride blood concentrations secondary to inhibition of cytochrome $4503 \mathrm{~A} 4$ metabolism by concomitant drug use of clarithromycin [14, 16], may contribute in clinics. Concomitant pharmacodynamic mechanisms related to additional blockade of cardiac potassium currents due to other drugs, disease states and electrolyte disturbances $[3,13]$ may cooperate. However, such interactions may reasonably be discounted in our patient.

The typical proarrhythmic response of antiarrhythmic drugs, particularly class III agents, but also of several noncardiac agents, is TdP which can develop in normal hearts but is more likely to occur in hypertrophied hearts, higher drug doses or tissue concentrations, female-gender, hypokalemia, hypomagnesemia and bradycardia with concomitant greater QT prolongation [1, 3, 7]. However, among 190 patients who presented nontraumatic VT out-of- hospital cardiac arrest with a supraventricular perfusing rhythm and a measurable QT interval, 62\% had monomorphic VT, whereas $38 \%$ had polymorphic VT of whom $49 \%$ (19\% of total) presented with prolonged QTc and TdP [8]. Interestingly, patients with prolonged QTc were not more likely to have polymorphic VT [8]. On the other hand, monomorphic VT and prolonged QTc has long been associated with agents otherwise known to increase the risk of TdP, such as clarithromicin [9], cesium chloride [10] and dofetilide [11]. These cases demonstrate that astute clinicians must be alert to the appearance of proarrhythmia with a variety of agents if the electrophysiological milieu is altered alongside of the possibility that an atypical presentation may coexist with monomorphic rather than polymorphic (TdP) VT [11]. Of note, our patient had no ventricular hypertrophy.

Different drugs (digoxin, diuretics and acenocumarol) taken by our patient might have contributed, yet this appears unlikely, to increase trimebutine toxicity (represented by QT prolongation and monomorphic VT). Although creatine clearance was normal in our patient, mitral valve disease with AF might have had a role. It has been proposed, reporting dofetilide-induced monomorphic VT in a 41-yearold male about a decade postmitral valve replacement for rheumatic disease, with pre-drug normal QTc and AF, that the proarrhythmia mechanism might have been a druginduced prolongation of refractoriness locally in diseased ventricular tissue, that could have impaired conduction such that the resulting inhomegeneity of repolarization and/or myocardial conduction produced a substrate for reentry [11]. Nevertheless, all this remains in the speculation domain and it is far from clear whether this could apply to explain proarrhythmia in our case.

A toxic effect of trimebutine seems crucial here. Indeed, the autonomous drug regimen $(1.350 \mathrm{mg} /$ day, the double of maximal recommended doses [19]) increase a week before emergency admittance and the normalisation of QT interval 
at drug discontinuation (from $523 \pm 12$ to $420 \pm 10 \mathrm{~ms}$, $\mathrm{p}<0.001$, compare Figs. 2 and 3 ), constitute a strong argument since the close relationship among these events makes them improbable as simple coincidences. Therefore, although there is a lack of causative relation among trimebutine increased regimen, QT prolongation and monomorphic VT in our patient, who had an otherwise not completely normal heart, but normal hemodynamics and normal coronary arteries, yet presented with $\mathrm{AF}$, we call attention to such an atypical presentation of drug-related proarrhythmia [11], probably due to toxicity. Whether this is due to an effect on both $\mathrm{I}(\mathrm{Kr})$ and $\mathrm{I}(\mathrm{Ks})$, as reported in guinea-pig ventricular tissue [18], deserves further investigation. Human myocardial tissues [2] may be appropriate for these studies, preferably concentration- and frequency-related.

It is also important to consider that in the rabbit, trimebutine exerts a $\mathrm{Ca}^{++}$channel blocking effect on the sinus node pacemaker cells, since above $10 \mu \mathrm{mol} / \mathrm{L}$ produced a negative chronotropic effect accompanied by decreases in $\mathrm{V}_{\max }$, slope of phase 4 depolarization and APA. The effects on the current systems were depression of the slow inward current and a decrease in the current oscillations induced by elevating external calcium [17]. On the other hand, in the guinea pig isolated papillary muscles, trimebutine maleate showed a depressant action on the electrical activities of the fast- and slow-response fibres of the heart, mainly due to inhibitions of both fast $\mathrm{Na}^{+}$and slow $\mathrm{Ca}^{++}$channels, since, always above $10 \mu \mathrm{mol} / \mathrm{L}$, decreased $\mathrm{V}_{\max }$ and $\mathrm{APD}_{90}$, whereas the resting potential was not significantly altered. Trimebutine also depressed the slow action potentials of papillary muscles produced by $27 \mathrm{mmol} / \mathrm{L} \mathrm{K}^{+}$and 0.2 $\mathrm{mmol} / \mathrm{L} \mathrm{Ba}^{++}$. Finally, in spontaneously beating sino-atrial node preparations, trimebutine (above $10 \mu \mathrm{mol} / \mathrm{L}$ ) decreased the heart rate, Vmax and the rate of diastolic depolarization [20]. The effect of trimbutine on heart rate might cooperate to increase QTc in man. However, our patient had AF, although average heart rate was slower while on trimebutine (Fig. 2: RR $=840 \mathrm{~ms}$ ) as compared to the ECG at discontinuation (Fig. 3: $\mathrm{RR}=720 \mathrm{~ms}$ ). With the difficulties of measuring and correcting QT in presence of AF, these point need be further considered and elucidated in future research.

Proarrhythmia is the adverse effect that most frequently causes the withdrawal of drugs marketed either as antiarrhythmics or for other uses [1-3]. This reflects the uncertainty of preclinical studies in detecting this risk, mainly mediated by the ability to block potassium channels. In clinical practice it can be quite difficult to establish a definitive diagnosis of proarrhythmia in a particular patient. In the case of non-antiarrhythmic drugs causal association is established when an effect on cardiac ion currents, primarily potassium currents, is shown at experimental level, with the contribution to clinical QT elongation that, in most cases, leads to pause-dependent polymorphic tachycardia on predisposed people with a prior shorter QT interval. Although this case meets only some of the features mentioned above, and the causal association between the occurrence of arrhythmia and the use of high doses of trimebutine is only probable, the absence of prior similar descriptions makes it of interest, which may prompt recognition of further cases. Electrophysiological studies might help clarifying these cases and might become indicated whenever other cases are reported. To clarify the effects of trimebutine on heart rate and QT in man, it might be of importance to have ECG measured before, during and after drug regimen in the gastroenterological milieu. Due to well-known longer average QT in women, sex- specific grouping is warranted.

\section{REFERENCES}

[1] Hohnloser, S.H. Proarrhythmia with class III anti-arrhythimc drugs: types, risk, and management. Am. J. Cardiol., 1997, 80, 82G-89G.

[2] Puddu, P.E.; Criniti, A.; Monti, F. Screening for drug-induced (acquired) long QT syndrome: is it time to apply new methods? (Editorial). Eur. Heart. J., 2001, 22, 363-369.

[3] Viskin, S.; Justo, D.; Zeltser, D. Drug induced prolongation of QT interval. N. Engl. J. Med., 2004, 350, 1013-1022.

[4] Lu, Z.; Kamiya, K.; Opthof, T.; Yasui, K.; Kodama, I. Density and kinetics of $\mathrm{I}(\mathrm{Kr})$ and $\mathrm{I}(\mathrm{Ks})$ in guinea pig and rabbit ventricular myocytes explain different efficacy of I(Ks) blockade at high rate in guinea pig and rabbit: implications for arrhythmogenesis in humans. Circulation, 2001, 104, 951-956.

[5] Ducroq, J.; Rouet, R.; Puddu, P.E.; Sallé, L.; Tabourel, C.; Ducouret, P.; Gérard, J.L. Electrophysiological effects of azimilide in an in vitro model of simulated-ischemia and reperfusion in guinea-pig ventricular myocardium. Eur. J. Pharmacol., 2005, 518, $165-174$.

[6] Drolet, B.; Khalifa, M.; Daleau, P.; Hamelin, B.A.; Turgeon, J. Block of the rapid component of the delayed rectifier potassium current by the prokinetic agent cisapride underlines drug-related lengthening of the QT interval. Circulation, 1998, 97, 204-210.

[7] Priori, S.G. Exploring the hidden danger of non-cardiac drugs. $J$. Cardiovasc. Electrophysiol., 1998, 9, 1114-1116.

[8] Brady, W.J.; De Behnke, D.J.; Laundrie, D. Prevalence, therapeutic response, and outcome of ventricular tachycardia in the out-ofhospital setting: a comparison of monomorphic ventricular tachycardia, polymorphic ventricular tachycardia, and torsades de pointe. Acad. Emerg. Med., 1999, 6, 609-617.

[9] Vallejo, C.N.; Rodríguez, P.D.; Sánchez, H.A.; Tornos M.M.P.; Ribera, E.; Soler, S.J. Ventricular tachicardia and long QT associated with clarithromycin administration in a patient with HIV infection. Rev. Esp. Cardiol., 2002, 55, 878-881.

[10] Dalal, A.K.; Harding, J.D.; Verdino, R.J. Acquired long QT syndrome and monomorphic ventricular tachycardia after alternative treatment with cesium chloride for brain cancer. Mayo. Clin. Proc., 2004, 79, 1065-1069.

[11] Reiffel, J.A. Atypical proarrythmia with dofetilide: monomorphic VT and exercise-induced torsade de pointe. Pace, 2005, 28, 877879.

[12] Criniti, A.; Picard, S.; Monti, F.; Dawodu, A.A.; Ruvolo, G.; Puddu, P.E.; Campa, P.P. Cicletanine prevents the excitationconduction blocks induced by terfenadine in ischemic myocardium. Eur. J. Pharmacol., 1999, 382, 197-205.

[13] Wysowsky, D.K.; Bacsany, J. Cisapride and fatal arrhythmia. $N$. Engl. J. Med., 1996, 335, 290-292.

[14] Sekkarie, M.A. Torsades de pointes in two chronic renal failure patients treated with cisapride and clarithromycin. Am. J. Kidney, Dis., 1997, 30, 437-439.

[15] Thomas, A.R.; Chan, L.N.; Bauman, J.L.; Olopade, C.O. Prolongation of QT interval related to cisapride-diltiazem interaction. Pharmacotherapy, 1998, 18, 381-385.

[16] van Haarst, A.D.; van't Klooster, G.A.; van Gerven, J.M. The influence of cisapride and clarithromycin on QT interval in healthy volunteers. Clin. Pharmacol. Ther., 1998, 64, 542-546. 
[17] Kotake, H.; Kinugawa, T.; Hirai, S.; Fukugi, M.; Hasegawa, J.; Mashiba, H. Effect of trimebutine maleate on sinus node pacemaker activity of the rabbit. Eur. J. Pharmacol., 1987, 144, 327-330.

[18] Morisawa, T.; Hasegawa, J.; Tanabe, K.; Watanabe, A.; Kitano, M.; Kishimoto, Y. Effects of trimebutine maleate on delayed rectifier $\mathrm{K}^{+}$currents in guinea-pig ventricular myocytes. J. Pharm. Pharmacol., 2000, 52, 403-438.
[19] Delvaux, M.; Wingate, D. Trimebutine: mechanism of action, effects on gastrointestinal function and clinical results. J. Int. Med. Res., 1997, 25, 225-246.

[20] Igawa, O.; Kotake, H.; Hirai, S.; Hisatome, I.; Hasegawa, J.; Mashiba, H. Effects of trimebutine maleate on electrical activities of isolated mammalian cardiac preparations. J. Pharm. Pharmacol., 1989, 41, 311-315.

(c) Schiariti et al.; Licensee Bentham Open.

This is an open access article licensed under the terms of the Creative Commons Attribution Non-Commercial License (http://creativecommons.org/licenses/by$\mathrm{nc} / 3.0 /$ ) which permits unrestricted, non-commercial use, distribution and reproduction in any medium, provided the work is properly cited. 